many conditions ascribed to infectious mononucleosis have been due to other viruses etc. The possible role of EB (Epstein-Barr) virus in the etiology remains to be further investigated. A proport on of seronegative cases develop EB antibodies and should probably be accepted as infectious mononucleosis. During preparation of the major part of the text further important observations on immunoglobulin levels, longterm cultures of leucocytes from infectious mononucleosis and the EB virus necessitated the addition of an appendix.

This summary of the present state of our knowledge is a valuable contribution and should act as a spur to further endeavour in this field. A better understanding of infectious mononucleosis may well provide the link between virus infections and neoplastic diseases.

\section{Pathology of Chronic Bronchitis and Emphysema}

Edited by Brian E. Heard. Pp. 136, illustrated. London: J. \& A. Churchill Ltd., 1969. 80s.

An analysis of the costs of medical care published by the Office of Health and Economics indicates that in England and Wales in 1967-68 the diseases of the respiratory system, including bronchitis, costs $£ 83.6$ million. Therefore, any book dealing with the common respiratory disease like bronchitis, is most welcome and this excellent monograph presents a study of structural changes occurring in the lungs of patients suffering from chronic bronchitis and emphysema. It is based on pathological investigations carried out over the last fourteen years in the Royal Postgraduate Medical School of London and in the University of Edinburgh.

The book is divided into four parts. The first one deals with Emphysema and occupies a major portion of the book. The second part deals with chronic bronchitis; the third with heart and other organs and the last one compares pathology with clinical radiology.

The book is very readable and the work is well presented and illustrated and except for the high price, it can be recommended to all doctors and medical students. A very useful list of references is given at the end of the book.

\section{Progress in Mental Health}

Edited by Hugh Freeman. Proceedings of the Seventh International Congress on Mental Health. Pp. xiv +346. London: J. \& A. Churchill, 1969. 60s.

Congresses and symposia multiply by the score, and it now seems obligatory to publish the proceedings, usually a long time after the event and in a variety of formats. This book is an edited version of the Proceedings of the Seventh International Congress on Mental Health held in London in August 1968. It was organized by the National Association for Mental Health on behalf of the World Federation for Mental Health and the main theme was mental health education. As always with the topic of mental health what is mostly discussed is in fact mental ill-health.

This gallimaufry is very uneven and for the most part hardly worth while putting into hard covers. The majority of the authors are from Western Europe or North America and although the Editor faced a formidable task, he does not make explicit his criteria for selection for publication. Some are mere snippets from work in progress or pilot studies, others generalizations from well known speakers, with a very variable amount of annotation and documentation.

The fifty contributions are divided into seven sections: (1) conflict, (2) social and cultural aspects, (3) public attitudes and education, (4) children and young people, (5) professional education, (6) clinical aspects and services, (7) theoretical aspects. There is a useful general index.
Man, Medicine and Morality

By A. E. Clark-Kennedy. Pp. 214. London: Faber \& Faber, 1969. $£ 2$.

In recent years the technical advances of medicine have received widespread publicity while relatively scant attention has been paid to the moral and legal implications of medical progress. It is timely, therefore, that many of the complexities of human medicine and the resultant problems facing doctors should now be brought into focus in a book both intended and suitable for the layman and for all whose work brings them into contact with medicine.

The opening chapters describe aspects of human physiology and psychology and the causation of organic disease and the functional disorders; they should be intelligible to the layman reader, but they contain a considerable number of errors. While some of the errors will not affect the overall comprehension, for example, the information that a woman's cells contain two $\mathrm{Y}$ chromosomes, others may cause confusion to the non-medical reader, for example, the transposition of the legends for the figures depicting the motor and sensory pathways. The statement that termination of pregnancy after the third month requires the removal of the uterus could result in unnecessary alarm and distress.

The first part of this book also acts as an introduction and background to the second which discusses the problems facing doctors in Britain at the present time and their relationship to society, the State, the law, and the church. The issues involved are set out clearly and without bias, giving the reader the opportunity to form his own opinions.

To write a book on medicine and its problems which will hold the interest of doctors and, in addition, be understandable to laymen requires a combination of medical and literary skill which few possess. Dr Clark-Kennedy has again demonstrated these skills. This book could be, and it is hoped will be, read with profit, enjoyment and enlightenment by many.

\section{Epidemiology of Cerebrovascular Disease}

Edited by John F. KurtzKe. Pp. 197. Berlin, Heidelberg, New York: Springer-Verlag, 1969. U.S. \$14.50.

This book describes most of the epidemiological facts of cerebrovascular disease known to the medical profession. It has an international flavour as it deals with incidence of the disease in different parts of the world, like Sweden, Norway, Denmark, Ireland, United States, South Africa and Japan.

An unusually large portion of the book (over 70 pages) is devoted to appendix, references and index, and the last chapter summarizes the previous twelve.

This book will not have an extensive sale. It is too expensive and its usefulness, if it exists at all, is very limited.

\section{Chromatographic and Electrophoretic Techniques, Vol. I Chromatography}

Third edition. Edited by I. Smith. Pp. $x i x+1080$. London: William Heineman Medical Books, 1969. 130s.

This book is a collection of experimentally biased papers on chromatographic techniques-by over thirty internationally established authors-and covers the major facets of the various relative fields. It is the third edition-the original appeared in 1957-and shows not only more extensive but also improved coverage which ranges over the qualitative aspects of paper, thin layer, and radioactive chromatographic techniques as well as certain quantitative facets and automation. Detailed methods covering ion exchange cellulose and Sephadex thin layer gel filtration are also given. Fractionation and sequence analysis of 32-P labelled ribonucleotides are included. Not only are standard procedures given but highly useful practical hints are detailed. A chapter on the investigation of new compounds is also included. The list 